\title{
MESURE DES GRADIENTS DE DENSITÉ DANS L'INTERACTION LASER-PLASMA
}

\author{
R. BENATTAR, C. POPOVICS, R. FABBRO, E. FABRE, C. GARBAN, \\ H. PÉPIN, A. POQUÉRUSSE, C. STENZ, J. VIRMONT et M. WEINFELD \\ Laboratoire de Physique des Milieux Ionisés, Groupe de Recherche du C. N. R. S. \\ Ecole Polytechnique, 91128 Palaiseau Cedex, France
}

\begin{abstract}
Résumé. - Nous étudions la densité électronique et son gradient dans l'interaction d'un laser à $\mathrm{CO}_{2}$ avec une cible plane de polyéthylène, pour un flux laser de $10^{12} \mathrm{~W} / \mathrm{cm}^{2}$. L'étude par interférométrie Jamin des profils de densité et la mesure strioscopique de son gradient, couplées à l'étude de l'image en rayons $X$ du plasma conduisent à une valeur de $220 \pm 80 \mu \mathrm{m}$ de la longueur du gradient de densité. Ceci est confirmé par des mesures indirectes obtenues à partir du bilan optique de l'interaction et du seuil de génération de l'harmonique $3 / 2 \omega_{0}$ du laser à $\mathrm{CO}_{2}$. Les conséquences de ces résultats sur les conditions de l'interaction sont discutées.
\end{abstract}

\begin{abstract}
We study the electron density and its gradient during the interaction of a $\mathrm{CO}_{2}$ laser with a polyethylene slab for a laser flux of $10^{12} \mathrm{~W} / \mathrm{cm}^{2}$. The Jamin interferometric study of density profiles and Schlieren measurements of its gradients, associated with the study of the $\mathrm{X}$-ray imaging of the plasma lead to a value of $220 \pm 80 \mu \mathrm{m}$ for the density gradient length. This is confirmed by indirect measurements obtained from the optical balance of the interaction and the threshold of $3 / 2 \omega_{0}$ harmonic of the $\mathrm{CO}_{2}$ laser. The consequences of these results on the interaction conditions are discussed.
\end{abstract}

1. Introduction. - La mesure de la densité électronique et de son gradient dans les plasmas créés par irradiation laser d'une cible solide est un élément important pour la connaissance du dépôt de l'énergie laser et des mécanismes d'interaction. En effet, parmi les processus d'absorption, le Bremsstrahlung inverse qui semble être le mécanisme prépondérant à bas flux dépend fortement de la longueur caractéristique du gradient. Dans ce cas, pour un profil de densité linéaire, le coefficient de réflexion du plasma prend la forme suivante :

$$
R=\exp \left[-5,46 T_{\mathrm{e}}^{-3 / 2} L\right]
$$

la température électronique $T_{\mathrm{e}}$ étant en $\mathrm{eV}$, la longueur de gradient $L$ en $\mu \mathrm{m}$.

Cette expression montre une dépendance exponentielle en fonction de la longueur de gradient.

Il a été montré [1] que l'absorption résonnante peut jouer aussi un rôle important dans le dépôt de l'énergie laser. Ce mécanisme présente un maximum d'efficacité pour une valeur $\theta$ de l'angle d'incidence donnée par la relation suivante :

$$
\left(k_{0} L\right)^{1 / 3} \sin \theta \simeq 0,8
$$

$\mathbf{k}_{0}$ étant le vecteur d'onde du faisceau incident et $L$ la longueur caractéristique du gradient de densité. Il apparaît ainsi que suivant l'ouverture de l'optique de concentration du faisceau et la valeur de $L$, l'absorption résonnante jouera un rôle plus ou moins grand.
La réfraction du faisceau laser dans le plasma est un mécanisme de perte très important qui est directement lié à la longueur du gradient de densité et à la forme du plasma. Et la détermination de ces paramètres est fondamentale pour estimer ces pertes.

Enfin, de nombreuses instabilités de chauffage (désintégration paramétrique ou deux plasmons) ou de rétrodiffusion (Brillouin ou Raman) peuvent se produire au cours de l'interaction. Le flux laser seuil à partir duquel elles apparaissent dépend fortement de la longueur caractéristique du gradient de densité. Par exemple, dans le cas de l'instabilité 2 plasmons qui engendre deux plasmons de fréquence $\omega_{0} / 2$, où $\omega_{0}$ est la fréquence laser incidente, le seuil est donné par la relation suivante [2] :

$$
I_{\mathrm{W} / \mathrm{cm}^{2}}=\frac{10^{14} T_{\mathrm{e}}(\mathrm{keV})}{600 L(\mathrm{~mm})} .
$$

Cette instabilité peut être mise en évidence par la génération de l'harmonique $3 / 2 \omega_{0}$ [3].

La longueur du gradient de densité est en principe déterminée par l'écoulement hydrodynamique du plasma. Cependant, des calculs théoriques récents [4] ont montré que les forces pondéromotrices associées à la pression de radiation du rayonnement laser peuvent conduire à une modification notable du gradient de densité. On peut ainsi obtenir un raidissement du profil de densité, en géométrie plane, et même la formation d'une bosse dans le cas d'une géométrie sphérique 


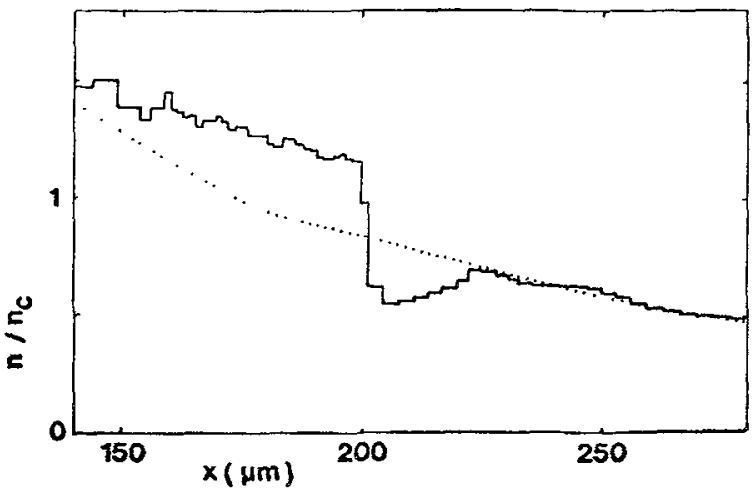

(a)

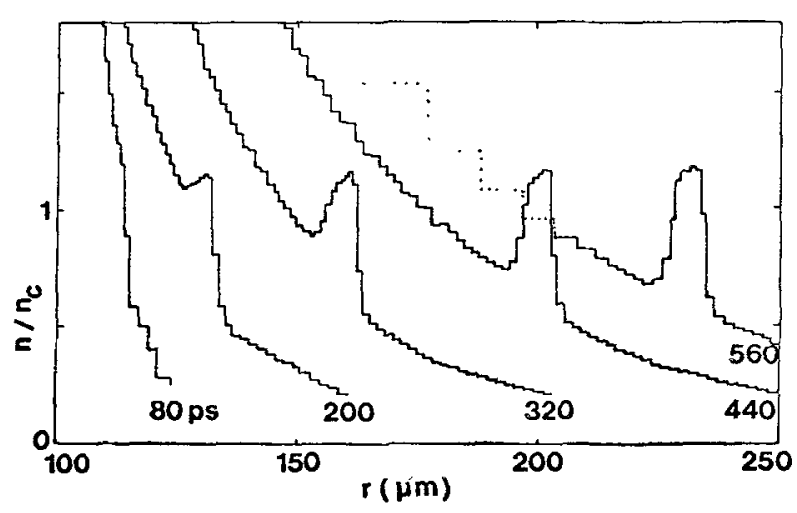

(b)

FIG. 1. - Modification du profil de densité sous l'effet de la pression de radiation (cible de DT, laser à néodyme, flux absorbé $\left.1014 \mathrm{~W} / \mathrm{cm}^{2} P_{\text {Laser }} / P_{\text {einétique }}=0,11\right): 1 a$ géométrie plane; $1 b$ géométrie sphérique.

(Fig. 1). Cet effet, qui peut modifier profondément les conditions de l'interaction, devient important lorsque la pression de radiation $P_{\mathrm{L}}$ est comparable à la pression cinétique $P_{\mathrm{c}}$ du plasma. Dans le cas de l'absorption résonnante, il faudra certainement en tenir compte.

Ces considérations nous ont conduits à mettre au point différents diagnostics du profil de densité qui sont, soit des mesures directes comme l'interférométrie et la strioscopie, soit des mesures indirectes comme la détermination du seuil de l'instabilité deux plasmons, le bilan optique de l'énergie laser réfléchie et l'imagerie en rayons $X$.

2. Description de l'expérience. - 2.1 Dispositif EXPÉRIMENTAL (Fig. 2). - On focalise au moyen d'un miroir sphérique, sur une cible plane de polyéthylène, le faisceau d'un laser à $\mathrm{CO}_{2}$, délivrant $40 \mathrm{~J}$ en 40 ns. Les dimensions de la tache focale ont été obtenues d'une part par l'étude des impacts du faisceau sur un papier thermosensible, et d'autre part par l'analyse de l'émission à $2 \omega_{0}$ en fonction du tirage du miroir. Ces deux méthodes fournissent un diamètre de tache focale de $250 \pm 50 \mu \mathrm{m}$. Cette valeur est proche de celle obtenue à partir de la photographie $X$ du plasma.

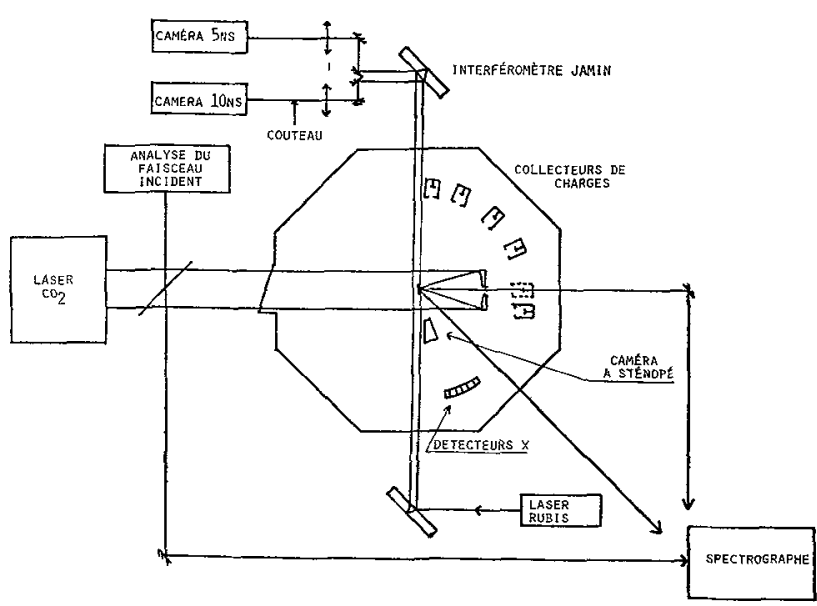

FIG. 2. - Dispositif expérimental.

(Fig. 9) qui donne un diamètre à mi-hauteur de $200 \mu \mathrm{m}$. L'intensité ainsi obtenue est de $10^{12} \mathrm{~W} / \mathrm{cm}^{2}$.

Les diagnostics mis en cuvre dans cette expérience sont les suivants :

- bilan optique de la lumière réfléchie à l'aide de photon-drags et de calorimètres ;

- spectroscopie de la lumière réfléchie à la fréquence $\omega_{0}$ et des harmoniques $3 / 2 \omega_{0}, 2 \omega_{0}, 3 \omega_{0}$ engendrés dans le plasma. Un spectroscope infra rouge type Ebert-Fastie, associé à des cellules $\mathrm{Hg}-\mathrm{Cd}$ Te permettent de reconstituer un spectre en une quarantaine de tirs lasers ;

- mesure de l'énergie des ions avec des collecteurs de Faraday ;

- mesure de température à partir du rayonnement $X$ par la méthode des absorbants;

- mesure résolue spatialement du rayonnement $X$ par chambre photographique à sténopé. Un sténopé de $60 \mu \mathrm{m}$ de diamètre est placé à $48 \mathrm{~mm}$ du plasma, son axe de visée faisant un angle de 0,1 rad avec la cible. La gamme d'énergie $X$ couverte est limitée par un filtre de béryllium de $11 \mu \mathrm{m}$ d'épaisseur. L'image est formée sur un film Kodirex dont on connaît la sensibilité absolue ;

- interférométrie Jamin et strioscopie décrits plus en détail ci-dessous.

\subsection{PRINCIPE DES MESURES INTERFÉROMÉTRIQUES} ET STRIOSCOPIQUes (Fig. 3). - Pour ces deux diagnostics, on utilise un faisceau sonde de fréquence de cou pure la plus faible possible, afin de sonder les densités électroniques les plus fortes possibles [5]. Dans le cas présent d'un plasma créé par un laser à $\mathrm{CO}_{2}$, l'utilisation d'un laser à rubis ( $1 \mathrm{~J}, 20 \mathrm{~ns}$ ) synchronisé au laser principal permet de sonder des régions de densité $\left(n_{\mathrm{e}}=10^{21} \mathrm{~cm}^{-3}\right)$ supérieure à la densité critique du laser à $\mathrm{CO}_{2}\left(n_{\mathrm{e}}=10^{19} \mathrm{~cm}^{-3}\right)$ [6]. Le montage est commun jusqu'à la sortie de l'interféromètre, ce qui permet de bien corréler les deux mesures qui s'effectuent suivant le même axe de visée à travers le plasma. 


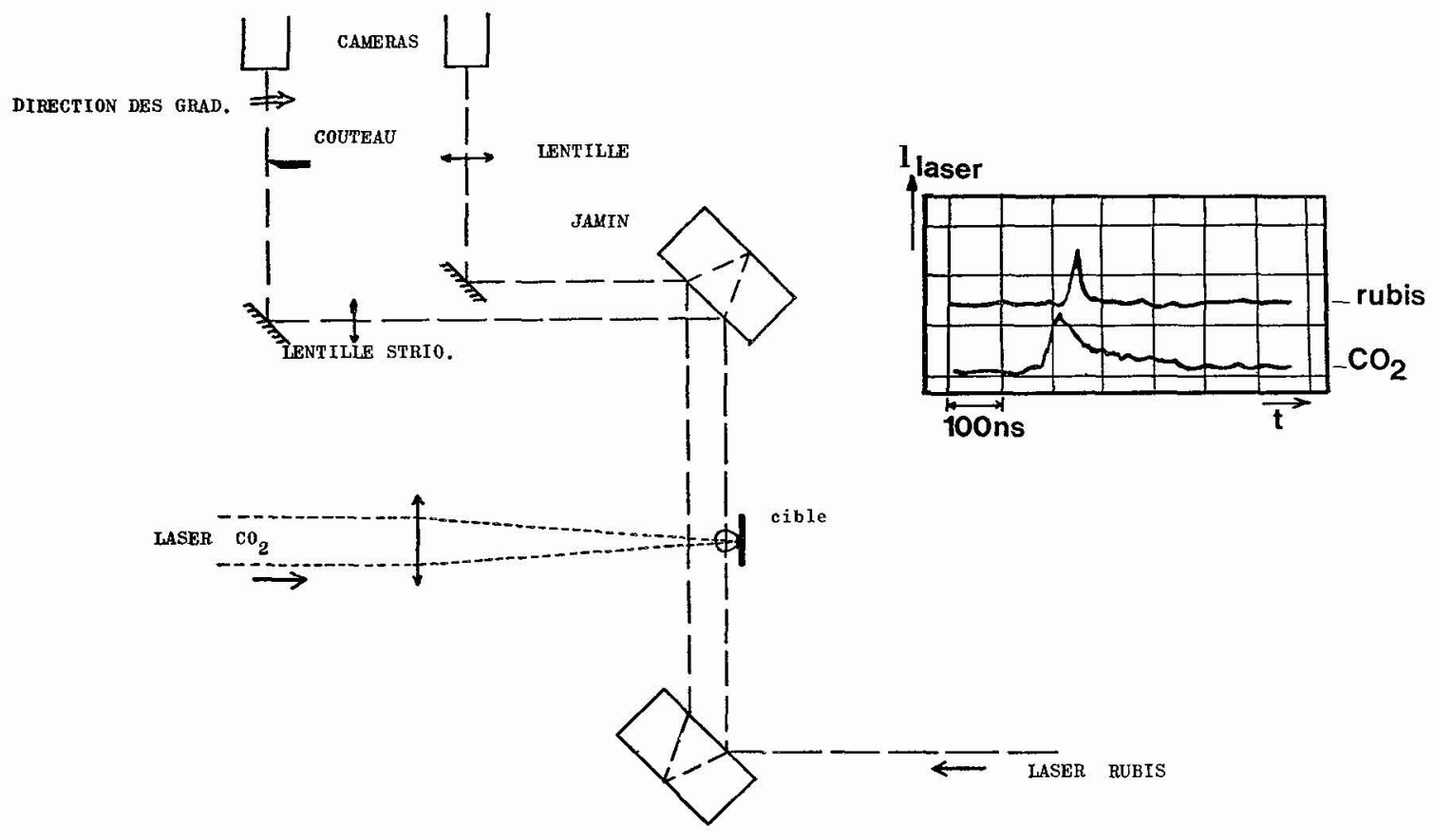

FIG. 3. - Montage interférométrique et strioscopique.

Flux laser : $10^{12}$ W. $\mathrm{cm}^{-2}$

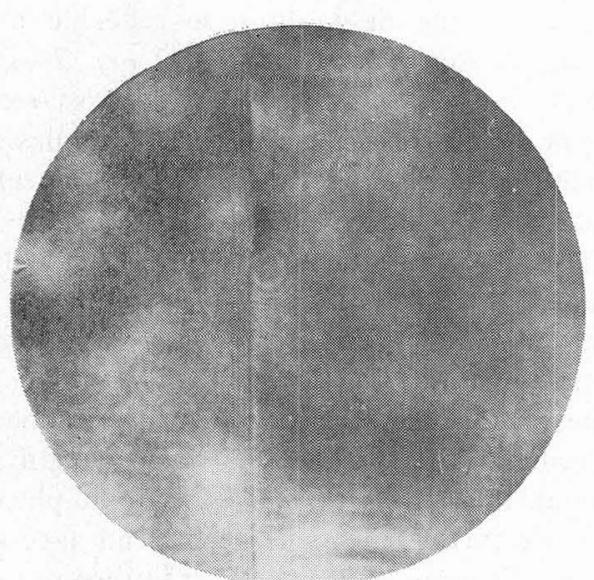

$-30 \mathrm{~ns}$

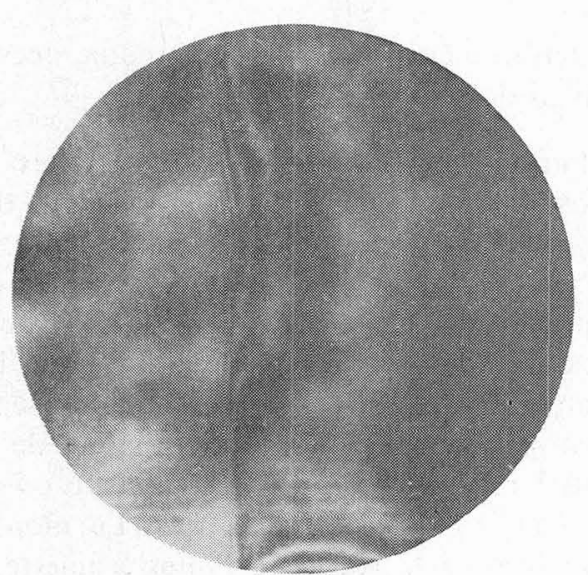

$+30 \mathrm{~ns}$

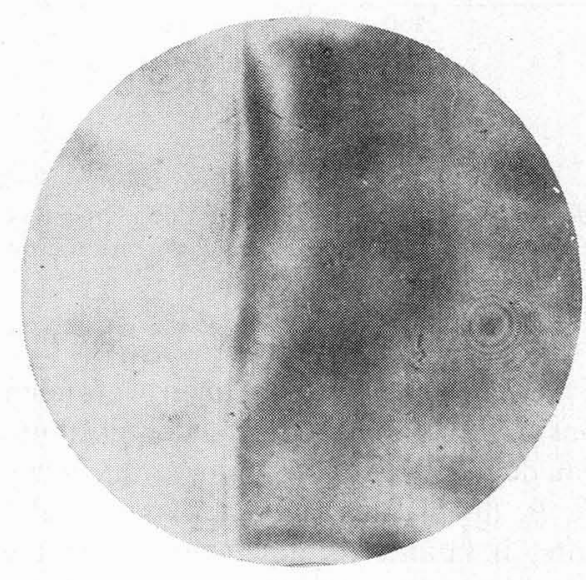

$0 \mathrm{~ns}$

$1 \mathrm{~mm}$

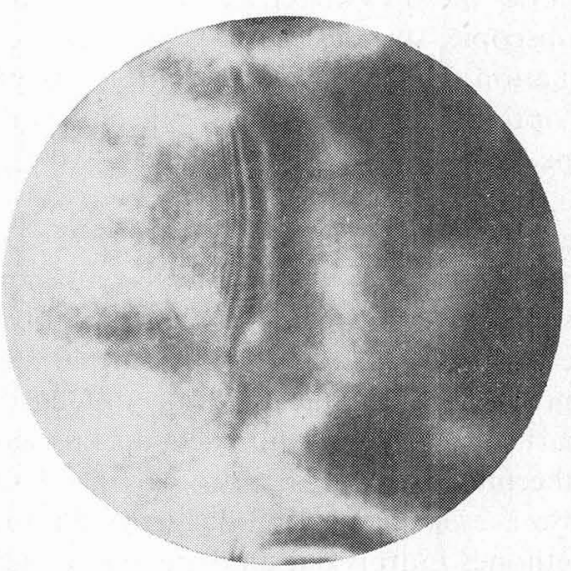

$+70 \mathrm{~ns}$

FIG. 4. - Interférogrammes du plasma à différents instants. 
Deux faisceaux sont réfléchis sur la lame de sortie de l'interféromètre : l'un est la recombinaison du faisceau de référence avec le faisceau passant sur le plasma et transporte l'information interférométrique. L'autre faisceau n'est pas recombiné au faisceau de référence et peut donc être utilisé pour la mesure strioscopique.

L'image interférométrique est obtenue sur une caméra électronique de temps de pose $5 \mathrm{~ns}$. A partir des interférogrammes, on remonte à la densité électronique par inversion d'Abel en supposant une symétrie axiale pour le plasma [7].

Le faisceau utilisé pour la mesure strioscopique est focalisé sur un couteau placé perpendiculairement au faisceau du laser à rubis et qui permet de sélectionner uniquement les rayons réfractés par les gradients perpendiculaires à la cible. L'image strioscopique $d u$ plasma est enregistrée par une caméra électronique de temps de pose $10 \mathrm{~ns}$. La divergence du laser à rubis et l'ouverture de l'optique permettent de mesurer des angles de déviation du faisceau dans le plasma compris entre $3 \times 10^{-4}$ rad et $3,9 \times 10^{-2}$ rad.

Pour ces deux mesures, l'historique de l'évolution du plasma est obtenu tir par tir en enregistrant à chaque tir le retard entre le laser sonde (synchrone de l'ouverture des caméras) et le laser à $\mathrm{CO}_{2}$ (Fig. 3).

3. Mesures interférométrique et strioscopique. 3.1 INTERFÉROMÉTRIE. - - La figure 4 montre l'évolution du plasma en fonction du temps, l'instant 0 étant pris au sommet de l'impulsion du laser à $\mathrm{CO}_{2}$. On peut noter que l'expansion du plasma est plus importante parallèlement à la cible que perpendiculairement : au maximum d'évolution du plasma situé environ $50 \mathrm{~ns}$ après le sommet de l'impulsion laser, le plasma a un diamètre de $2 \mathrm{~mm}$ et une épaisseur de $500 \mu \mathrm{m}$ environ [8]. Il faut noter la présence de quelques franges de diffraction sur le bord de la cible, qui introduisent une erreur systématique d'environ $80 \mu \mathrm{m}$ dans la détermination de la position de la cible, et également dans le calcul de la densité électronique au voisinage de la cible.

La figure $5 a$ montre les profils de densité obtenus à partir de l'interférogramme enregistré $30 \mathrm{~ns}$ après le sommet de l'impulsion laser. Les parties pointillées représentent la zone d'indétermination due aux franges de diffraction. Ces profils montrent que la densité critique est localisée sur la surface de la cible à $100 \mu \mathrm{m}$ près. La figure $5 b$ donne une longueur minimale du gradient de densité de $180 \mu \mathrm{m}$. Le dépouillement d'un autre tir laser, au même instant, conduit à une valeur de $300 \mu \mathrm{m}$.

3.2 Mesures StrioscopiQues. - La figure 6 représente les striogrammes du plasma à des temps différents pour deux positions du couteau :

- la planche (a) est un enregistrement à la sensibilité maximale du système. Les angles de déviation admis sont supérieurs ou égaux à $3,1 \times 10^{-4} \mathrm{rad}$;

- la planche $(b)$ correspond à un enregistrement sélectionnant des angles $\alpha \geqslant 7,7 \times 10^{-4} \mathrm{rad}$.

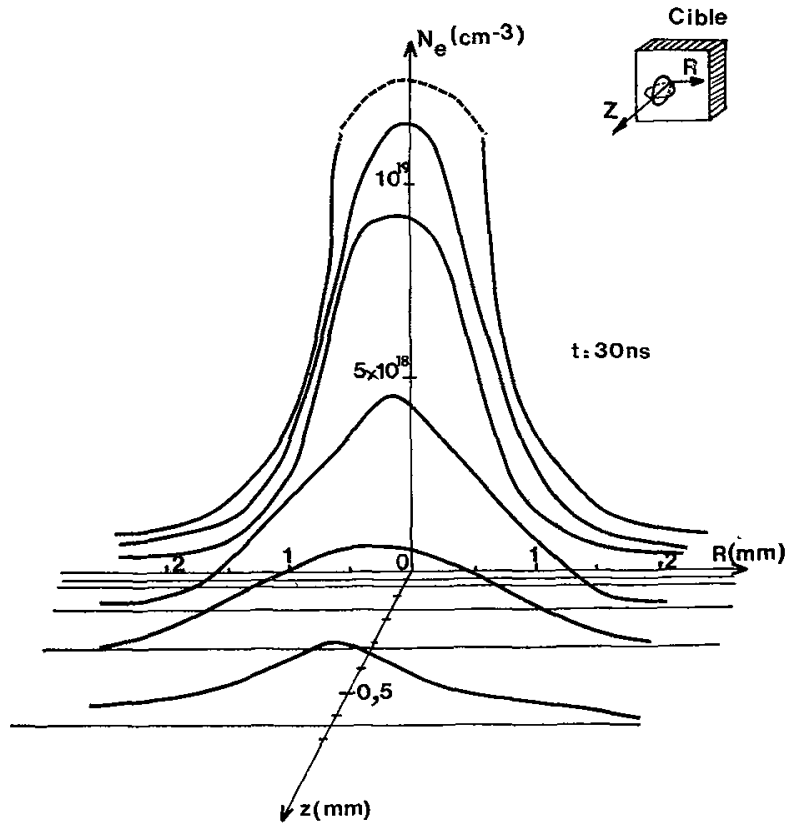

(a)

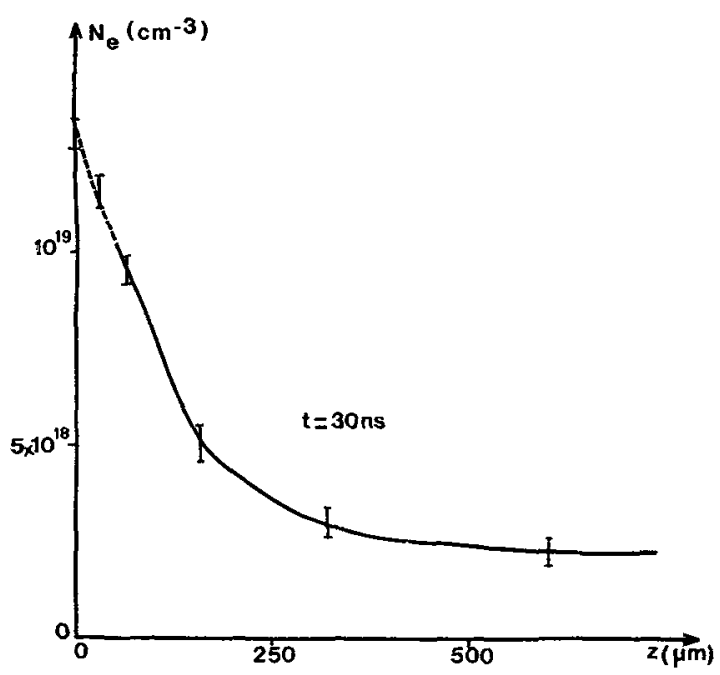

(b)

FIG. 5. - Profils de densité : $5 a$ représentation à trois dimensions de la densité du plasma ; 5 b profil de densité le long de l'axe du plasma.

Tous les enregistrements ont été pris par superposition de deux photos, d'abord sans plasma pour avoir la position de la cible seulement, ensuite avec plasma.

La comparaison des planches $a$ et $b$ montre que dans le front du plasma, la déviation des faisceaux réfractés est comprise entre 3,1 et $7,7 \times 10^{-4} \mathrm{rad}$. Par contre, une zone à gradients plus forts $\left(\alpha>7,7 \times 10^{-4} \mathrm{rad}\right)$ est observée près de la cible et s'en détache au cours de l'évolution du plasma pendant $50 \mathrm{~ns}$, puis s'en rapproche. La figure 7 qui donne la position d'un front de gradient correspondant à $\alpha \geqslant 7,7 \times 10^{-4}$ rad montre plus précisément ce mouvement. 


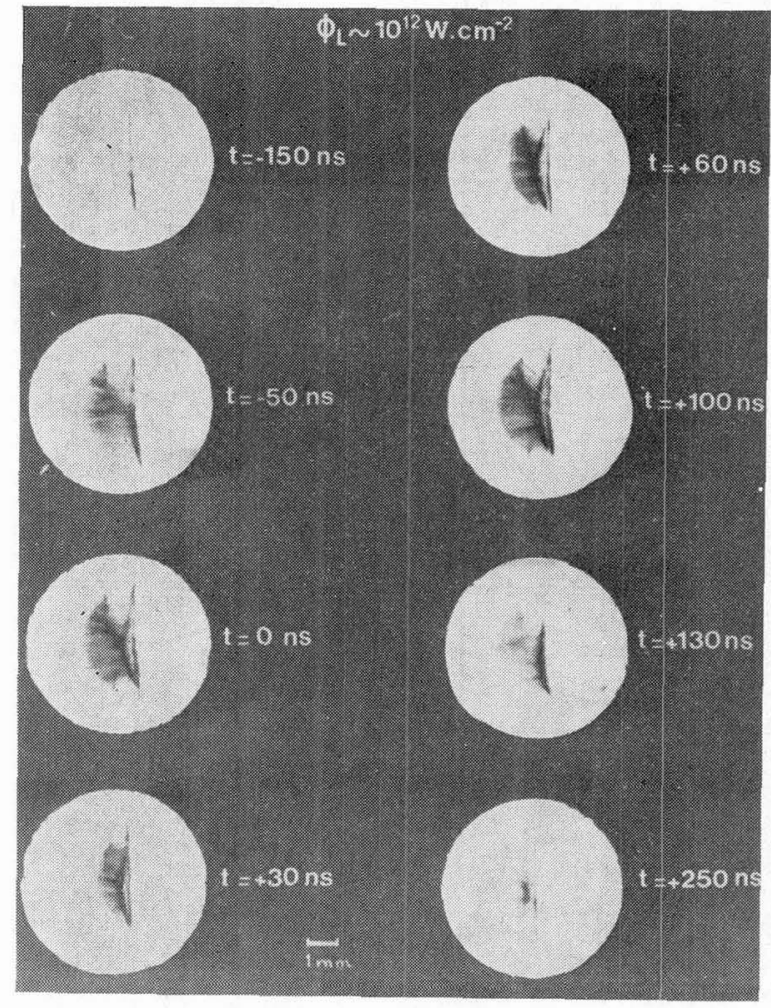

Planche (a)

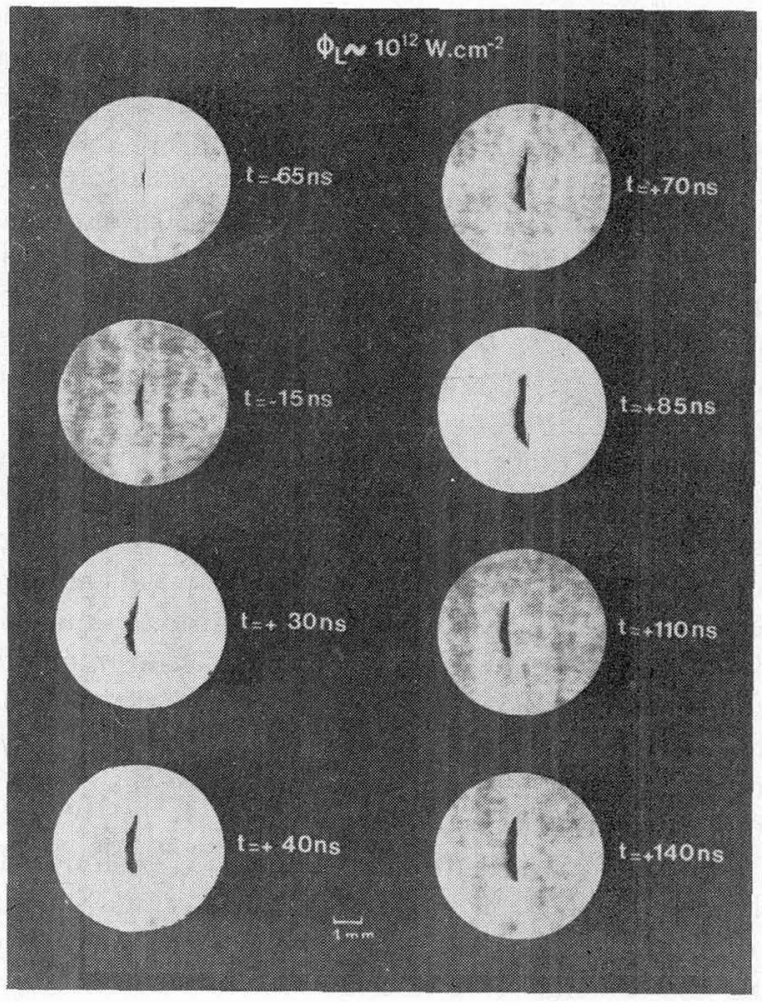

Planche (b)

Fıg. 6. - Striogrammes du plasma à différents instants : $6 a$ angles de déviation sélectionnés supérieurs à $3 \times 10^{-4} \mathrm{rad}$; $6 b$ angles de déviation sélectionnés supérieurs à $7,7 \times 10^{-4}$ rad.

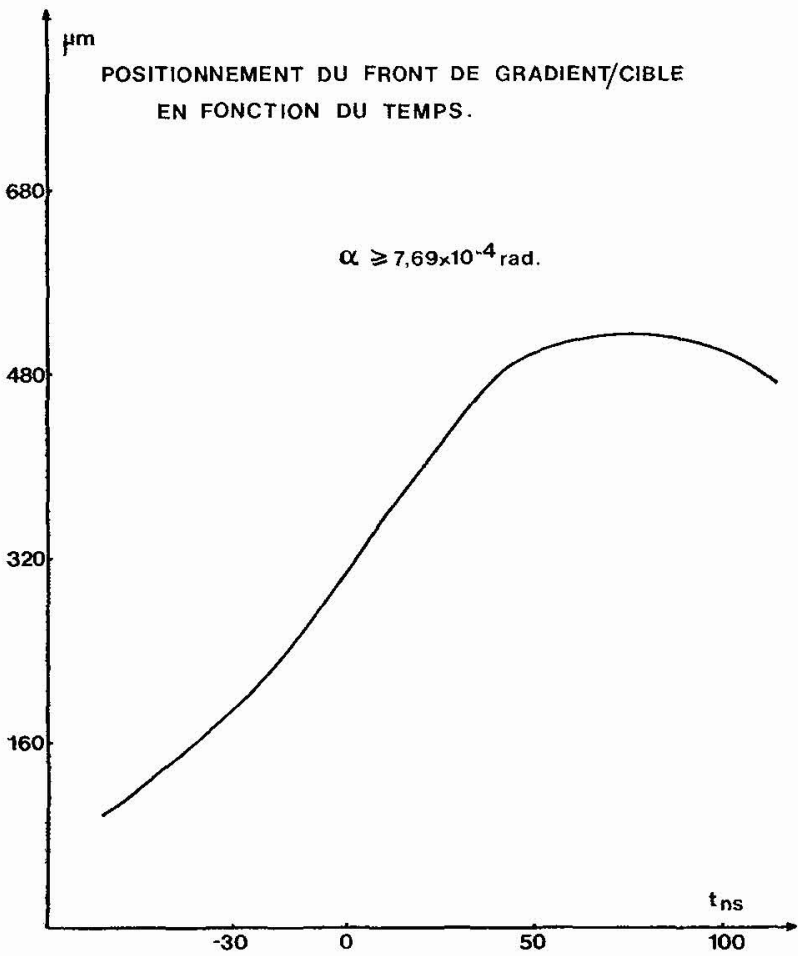

FIG. 7. - Position du front de gradient en fonction du temps, pour les angles sélectionnés supérieurs à $7,7 \times 10^{-4} \mathrm{rad}$.
Pour des angles sélectionnés supérieurs à $7,7 \times 10^{-4} \mathrm{rad}$, cette zone lumineuse garde ses dimensions et ne disparaît que pour un angle

$$
\alpha>9,1 \times 10^{-3} \mathrm{rad} \text {. }
$$

Ceci détermine la valeur maximale des gradients dans le plasma. Pour un plasma de $1 \mathrm{~mm}$ de diamètre et dans l'hypothèse d'un gradient normal à la cible, on obtient une valeur du gradient de $4,2 \times 10^{20} \mathrm{~cm}^{-4}$, ce qui correspond au voisinage de la densité critique à une valeur de la longueur de gradient de $230 \mu \mathrm{m}$. Dans l'hypothèse d'un plasma à symétrie radiale, de $1 \mathrm{~mm}$ de rayon, la valeur du gradient serait de $7 \times 10^{20} \mathrm{~cm}^{-4}$ et sa longueur caractéristique $140 \mu \mathrm{m}$.

Dans un modèle de ce type, les rayons très proches de la cible subissent une très faible déviation quelle que soit la valeur du gradient de densité. Ces rayons sont alors arrêtés dans tous les cas par le couteau. Cet effet pourrait expliquer l'existence d'une zone sombre au voisinage de la cible (Fig. 6). Il faut remarquer que cette zone sombre ne peut pas être expliquée par la présence de gradients très raides faisant sortir les rayons lumineux de l'optique de collimation car cet effet n'est pas visible en ombroscopie.

4. Mesures indirectes des gradients de densité, 4.1 BILAN OPTIQUE. - Le bilan optique $[9,10]$ montre 
que $7 \%$ environ de l'énergie laser est réfléchie dans le plasma dans les conditions de l'expérience. Si l'on suppose que la majeure partie de cette absorption est due au mécanisme de Bremsstrahlung inverse, la comparaison des prévisions théoriques données par la relation (2) à la valeur mesurée pour l'absorption conduit à une mesure indirecte de la longueur de gradient [9]. En prenant la température électronique égale à $300 \mathrm{eV}$ comme le montrent les mesures de rayonnement $X$ [11], on estimerait ainsi la longueur du gradient moyen de densité entre 350 et $400 \mu \mathrm{m}$.

4.2 HARMonique $3 / 2 \omega_{0}$. - L'harmonique $3 / 2 \omega_{0}$ [3-10] étant engendrée par l'instabilité 2 plasmons suivie d'une reconversion Raman d'un plasmon à $\omega_{0} / 2$ avec un photon incident, son émission présente le même seuil que l'instabilité 2 plasmons. La mesure de seuil (Fig. 8), associée à la mesure de la température du plasma permet d'évaluer la longueur du gradient de densité. Le flux seuil étant estimé entre 1 et $2 \times 10^{11} \mathrm{~W} / \mathrm{cm}^{2}$ et la température associée à ce flux étant de $170 \mathrm{eV}$, on en déduit une longueur de gradient $n_{\mathrm{c}} / 4$ de $150 \mu \mathrm{m}$. Il faut cependant mentionner que la détermination du gradient par cette méthode est obtenue pour un flux égal au seuil de l'instabilité et donc plus faible que dans les autres mesures.

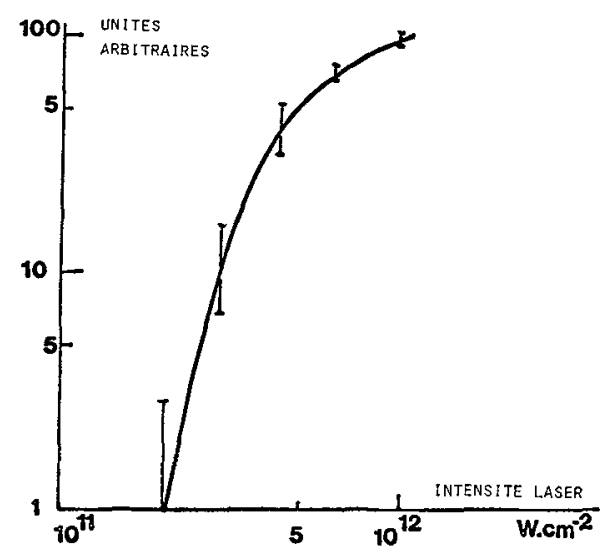

FIG. 8. - Génération de l'harmonique $3 / 2 \omega_{0}$.

4.3 IMAGE EN RAYONS X. - L'image obtenue en rayons $X$ permet de déterminer les dimensions de la zone émissive, parallèlement et perpendiculairement à la cible. La figure 9 présente deux densitogrammes, axial et radial, d'une image obtenue en 15 tirs. Le rayonnement recueilli comprend les rayonnements de freinage et de recombinaison, mais exclut les raies, qui ont toutes une énergie extérieure à la bande passante du filtre utilisé, dans les conditions expérimentales présentes.

Ces deux rayonnements continus croissent comme $n_{\mathrm{e}}^{2}$. Donc, si l'on suppose la température constante dans la zone d'interaction (hypothèse assez raisonnable, vu les données de codes hydrodynamiques), la longueur

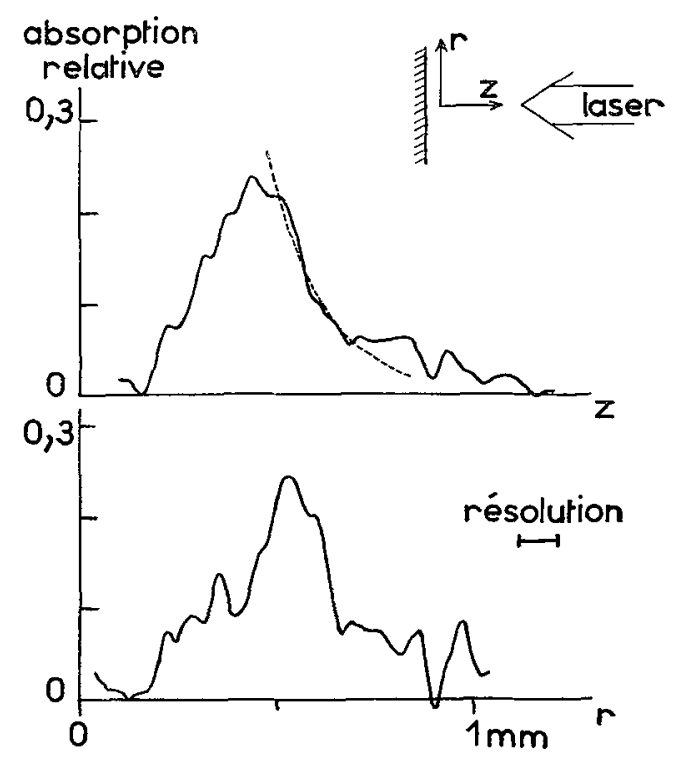

Fig. 9. - Microdensitogrammes d'une photographie du plasma en rayons $\mathrm{X}$. L'origine des abcisses est arbitraire.

caractéristique de décroissance de l'intensité d'émission $\mathrm{X}$ est proportionnelle à la longueur de décroissance de $n_{\mathrm{c}}^{2}$.

Le profil axial de la figure 9 donne en direction du laser une intensité décroissante assimilable à une exponentielle de longueur caractéristique $130 \mu \mathrm{m}$ (représentée en pointillé). Ceci conduit à une longueur de gradient double de cette valeur pour la densité électronique, soit $260 \mu \mathrm{m}$.

5. Discussion des résultats. Conclusion. -- Le principal résultat obtenu est une mesure du gradient de densité par différentes méthodes optiques : interférométrie, strioscopie et émission du rayonnement $X$. L'ensemble de ces valeurs est en bonne concordance et nous permet de déterminer une longueur caractéristique du gradient de $220 \pm 80 \mu \mathrm{m}$ dans la zone de densité critique. Cette valeur est en assez bon accord avec la détermination effectuée à partir du seuil de l'instabilité 2 plasmons.

Par ailleurs, nous n'avons pu mettre en évidence aucune modification de gradient due à l'effet des forces pondéromotrices dans cette expérience. Pour les valeurs de flux laser utilisées dans l'expérience présente, il est certain qu'on ne peut attendre un effet de ce type car la pression cinétique reste toujours supérieure à la pression de radiation.

Des expériences ultérieures, réalisées avec des flux supérieurs $\left(10^{13} \mathrm{~W} / \mathrm{cm}^{2}\right.$ en $\mathrm{CO}_{2}$ ou $10^{16} \mathrm{~W} / \mathrm{cm}^{2}$ en néodyme) devraient nous permettre, grâce aux diagnostics décrits ci-dessus, de vérifier l'existence de telles modifications.

Une autre conséquence de ces résultats réside dans l'interprétation du coefficient de réflexion mesuré : si 
l'on utilise la longueur de gradient déterminée expérimentalement, le coefficient de réflexion devrait être d'environ $80 \%$. Compte tenu de la valeur de $70 \%$ mesurée pour le coefficient de réflexion totale du plasma, ceci semble montrer que le Bremsstralhung inverse n'est pas le seul mécanisme qui régit l'absorption dans cette expérience. L'absorption résonnante peut dans ce cas avoir une contribution importante. En effet, l'angle optimal dans le cas présent est de 8 à $9^{\circ}$ et correspond à l'ouverture totale de l'optique de focalisation.

Remerciements. - Nous remercions MM. Maignan, Michard, Olmédo, Timsit et Thuilier pour leur assistance technique. Une partie de ces recherches a bénéficié du soutien de la D. R. M. E. Ces travaux ont été menés dans le cadre du programme scientifique $d u$ GRECO Interaction Laser-Matière.

\section{Bibliographie}

[1] Forslund, D. W., Kindel, J., Lee, K., Lindman, E. L., MORSE, R. L., Phys. Rev. A 11 (1975) 679.

[2] Rosenbluth, M. N., Phys. Fluids 17 (1974) 1211.

[3] Bobin, J. L., Decroisette, M., Meyer, B., Vittel, Y., Phys. Rev. Lett. 30 (1973) 594.

[4] Lee, K., Forslund, D. W., Kindel, J. M., Lindman, E. L., Phys. Fluids 20 (1977) 51.

Brugckner, K. A., JANDA, R. S., Nucl. Fusion 17 (1977) 451.

Virmont, J., Pellat, R., Mora, P., Waves and Instabilities in plasmas conference, Palaiseau, 4 D 3 (1977). Soumis à Phys. Fluids.

[5] Attwood, D. T., Coleman, L. W., Sweeney, D. W., Lawrence Livermore Laboratory report UCRL, 50021-75 (1975) et Appl. Phys. Lett. 26 (1975) 616.

[6] Engelhardt, A., Carman, R., Bull. Ann. Phys. Soc. 21 (1976) 1046.
Bocher, J. L., Elie, J. P., Martineau, J., Rabeau, M., Bull. Ann. Phys. Soc. 21 (1976) 1119.

Fedosejevs, R., Tomov, I. V., Rrchardson, M. C., Bull. Ann. Phys. Soc. 21 (1976) 1047.

[7] Schirmann, D., Grelot, P., Rabeau, M., Tonon, G., Rapport CEA R-4299 (1972).

[8] Tonon, G., Thèse d'état, Université de Grenoble (1973), p. 71.

[9] Garban, C., Thèse de $3^{e}$ cycle, Université Paris VI (1977).

Farre, E., Garban, C., Popovics, C., Poquerusse, A., Stenz, C., VIRMONT, J., Plamsa Physics and Controlled Nuclear Fusion Research IAEA, CN 35/G 3-4 (1976).

[10] Martineau, J., Bocher, J. L., Elie, J. P., Rabeau, M., European Conference on laser interaction, Palaiseau, C 8 (1976).

[11] Stenz, C., Popovics, C., Fabre, E., Virmont, J., Poquerusse, A., Garban, C., J. Physique 38 (1977) 761. 